s)ges stas

مرورى بر تركيبات F و اــقتوكينونى حاصل از جنس ديوسييروس

\author{
بهرام بعى باى'، حسين كمالى'، عليرضا نعمت اللهى"

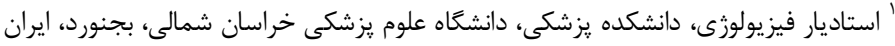

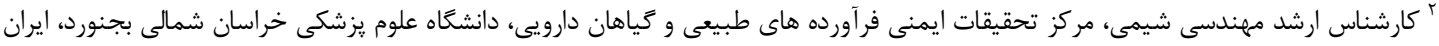

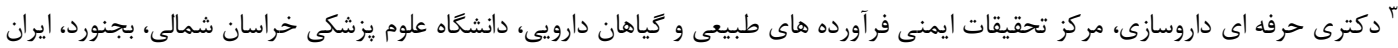

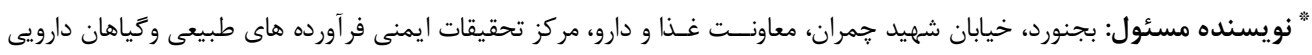

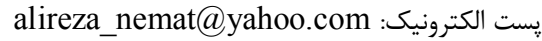

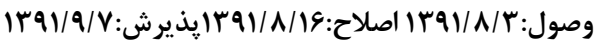

جكيده

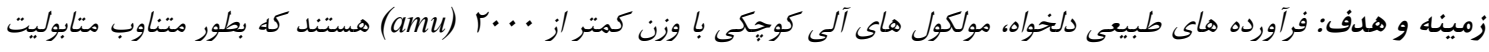

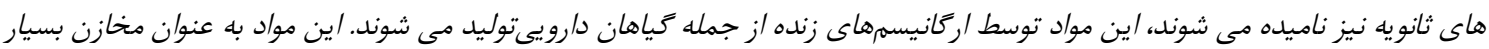

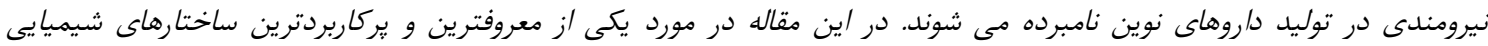

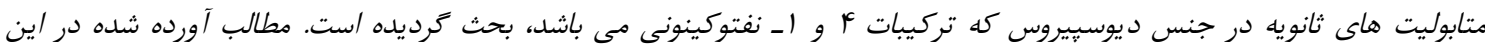

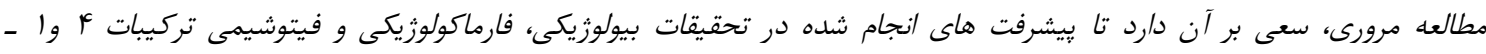

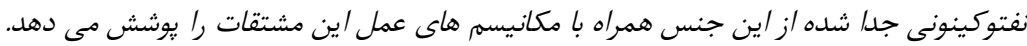

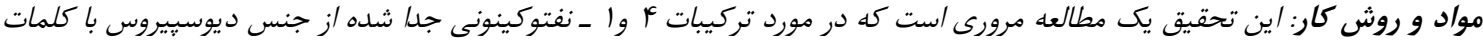

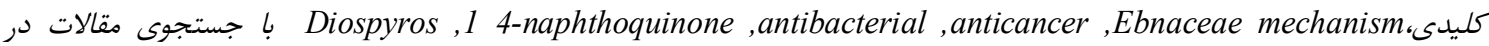

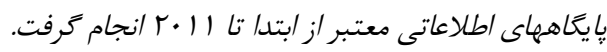

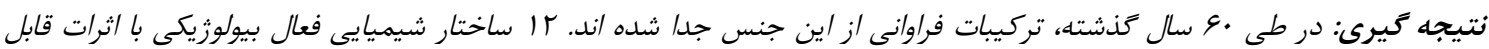

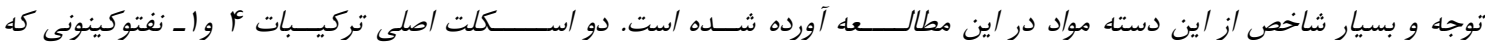

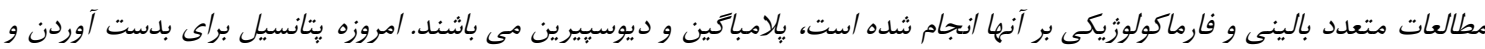

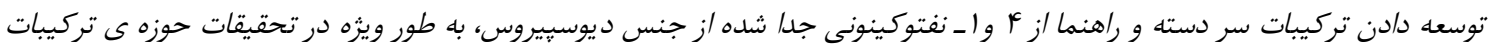

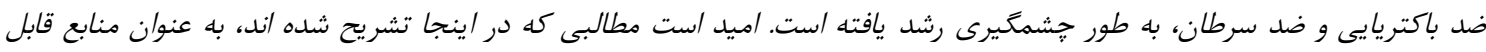

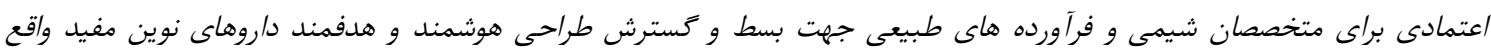
كردند. واثه هاى كليدى: ديوسيبروس، أ و ا - نفتوكينون، ضد باكتريايى، ضد سرطان، مكانيسه عمل

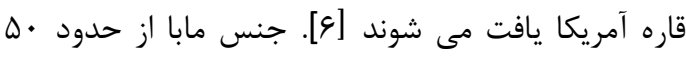
مقدمه كونه كه در جنوب شرق آفريقا يافت مى شوند، تشكيل فاميلى إبناسه كروك از شش جنس (ديوسيروس، اوسلا،

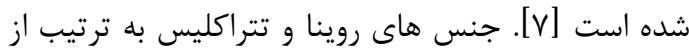

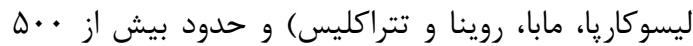

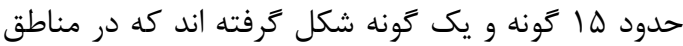

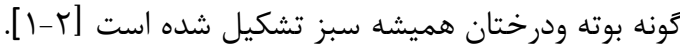

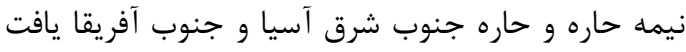

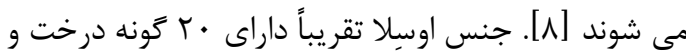
در بين تمامى اين جنس ها، بيشترين مطالعه بر روى

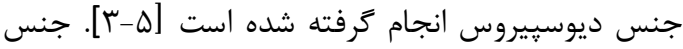

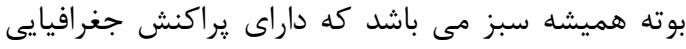
ليسوكاريا شامل هشت كونه مى باشد كه بيشترين آنها در 
اي از فنول هاى طبيعى مى باشنـــد كه داراى اســـكلت

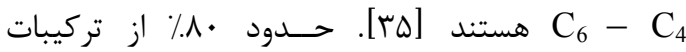



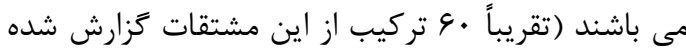

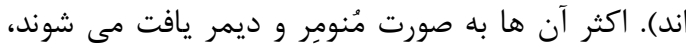

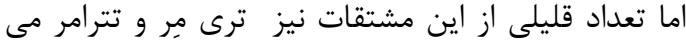

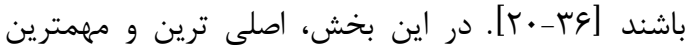

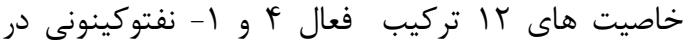
شكل ا شرح داده شده اند.

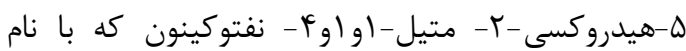
يلامبازين (1) شناخته مى شود براى اولين بار از كياه

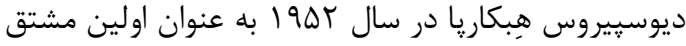

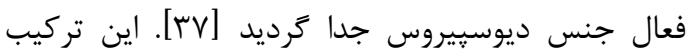

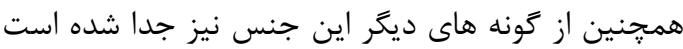

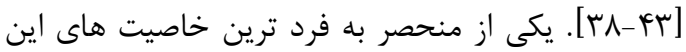

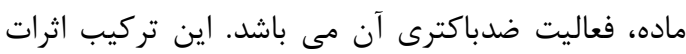

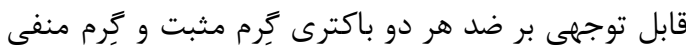

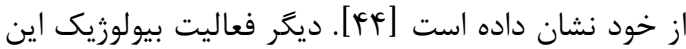

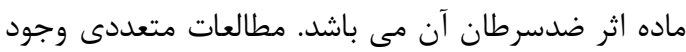

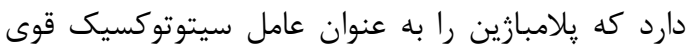

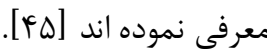

اين ماده همجنين اسكلت اوليه بسيارى از تركيبات

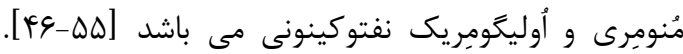

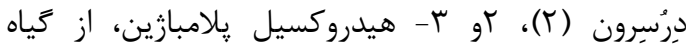
D. maritima Blume خاهت D. melanoxylon Roxb

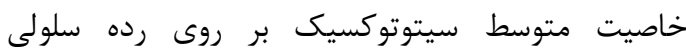
ا ا با MCF-7

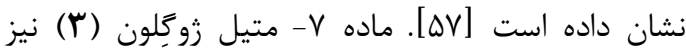
يكى از مهمترين اسكلت هاى مونومريك و اليكومريك ادهن

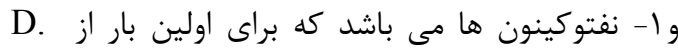


ساليان ديخر در كونه هاى متفاوت ترى نيز كشف شد

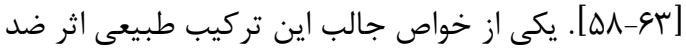

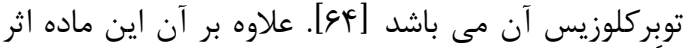

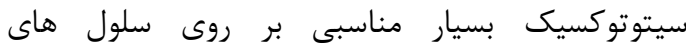
كارسينوماى بدخيم انسانى نشان داده است [بr -•r].
خوبى در جنوب آفريقا وجزاير كومور مى باشند. [9]

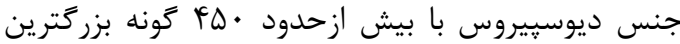

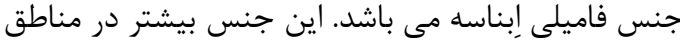

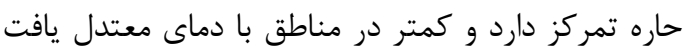

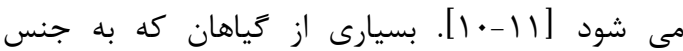
ديوسيروس تعلق دارند، در طب سنتى براى اهداف مختلف كاربرد دارند. مطالعات بيولوزيك و فارماكولوزيك ديك

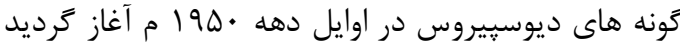

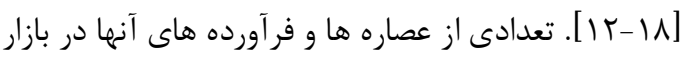

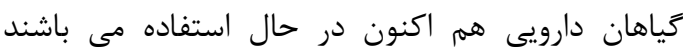

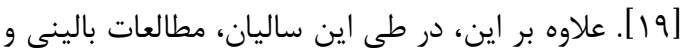

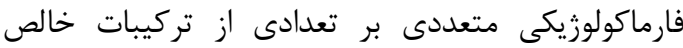

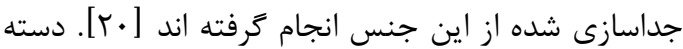
تركيب ع و و ا- نفتوكينونى يكى از ازين شاخص ترين ساختارهاى شيميايى جنس ديوسيِيروس مى باشئ همجنين طيف وسيعى از اثرات بيولوزيكى همجيحون، آثار

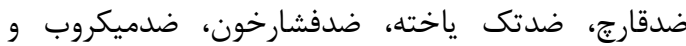

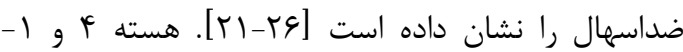

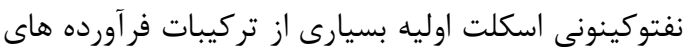

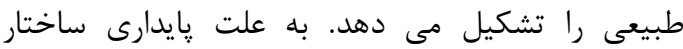
شيميايى اين دسته از تركيبات، فعاليت هاى ضد باكترى آندى و ضد سرطان قابل توجهى براى آنها شناسايى شده اند فائ فائ

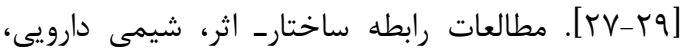
تكنيك هاى نوين مدلينَ كامييوترى و تكنيك هاى سنتزهاى نيمه مصنوعى اين دسته از تركيبات، براى

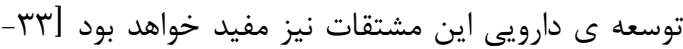

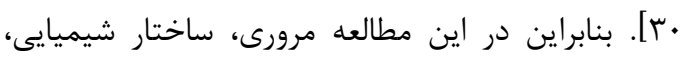

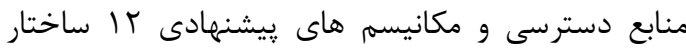
شناخته شده (شكل () از نظر بيولوزيكى و فارماكولوزيكى

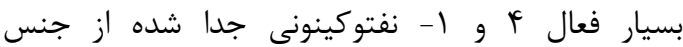
ديوسيّيروس در راستاى روشن شدن يتانسيل اين جنس

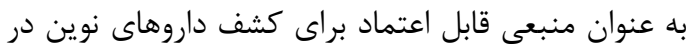
آينده بحث شده است.

\section{تركيبات بيولوزيك فعال f و أ- نفتوكينونى جنس

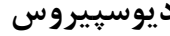

جنس ديوسيروس يكى منبع غنى از نفتوكينون ها مى دي دئ باشد [ץץ]. تمامى اين دسته از تركيبات متعلق به دسته 


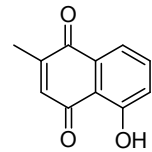

plumbagin (1)<smiles>Cc1cc(O)c2c(c1)C(O)CCC2=O</smiles>

Shinanolone (4)

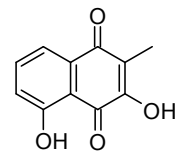

Droserone (2)

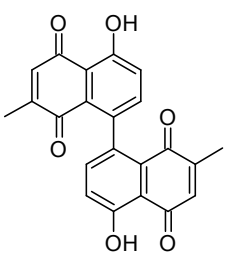

Maritinone (5)<smiles>Cc1cc(O)c2c(c1)C(=O)C=CC2=O</smiles>

7-Methyljuglone (3)

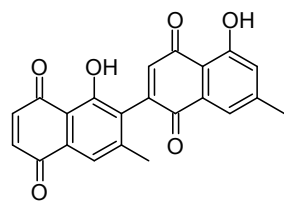

Diospyrin (6)

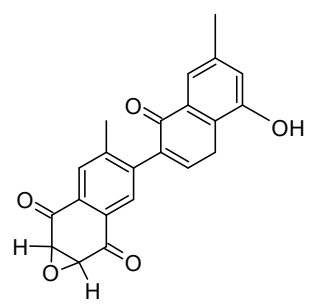

Diosquinone (8)

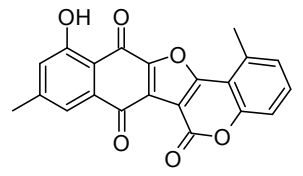

Crassiflorone (10)

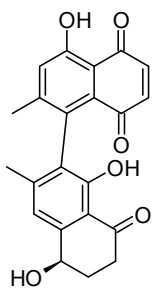

Isodiospyrol A (12)

4-hydroxy-3,5-dimethoxy-2-naphthaldehyde (11)

\section{شكل ا: ساختار شيميايى تركيبات اوf - نفتوكينونى}

يلامباكين مى باشد كه از گونه هاى D. martima، كاكى

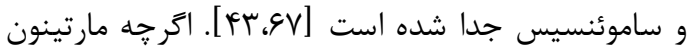

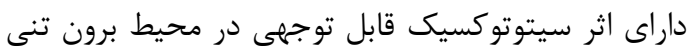
با كا IC كارآزمايى بالينى اثبات شده است كه در محيط درون تنى
شينانولون (Y) كه تركيب كاهش يافته V- متيل جاكلون مى باشد از گونه هايى نظير D. kaki Hiern، مينگايى،

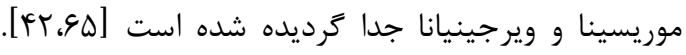
اين ماده به علت خاصيت منحصر به فرد ضدباكترى خود

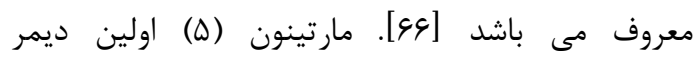




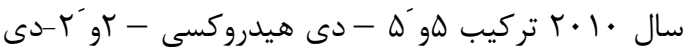

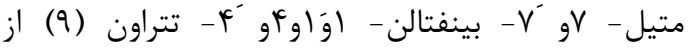

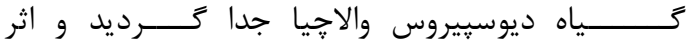

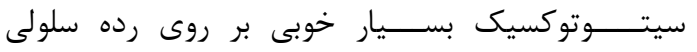

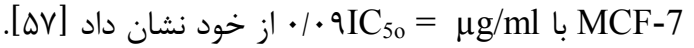
كراسى فلورون (•) از كونه كراسى فلورا جدا كرديد.

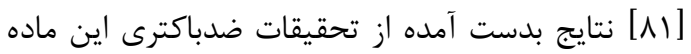

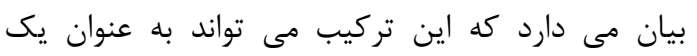
تركيب راهبر نوين براى اثرات ضدتوبر كلوزيس و ضد كنورآ باشد [AT].

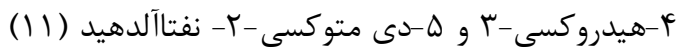
جدا شده از زونه آسيميليس براى اثرات ضديرتوزآ در محيط برون تن آزمايش شد. اين تركيب اثرات متوسطى لهى

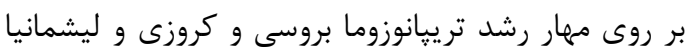
دونوانى به ترتيب با 19/1 IC IC و

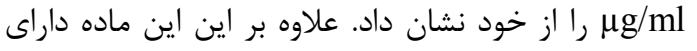

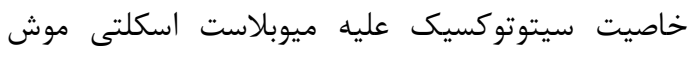
A صحرايى (سلول هاى L-6) مى باشد. ايزوديوسيرال (IT)، مشتقى از ايزوديوسييرن مى بـ باشد كه از كونه اريتيودِس جدا شده است. اين ماده داراى اثر ضدمالاريايى

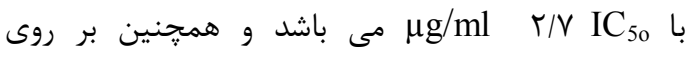

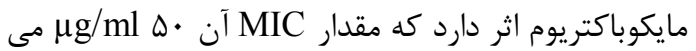
باشد. شايان ذكر است اين ماده بر روى رده هاى سرطان

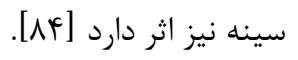

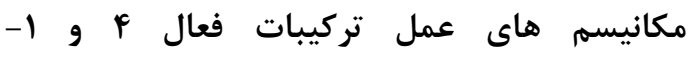
نفتوكينونى بسيارى از تركيبات أل و ا - نفتوكينونى جدا شده از جنس

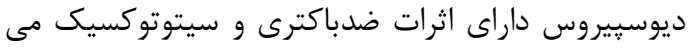

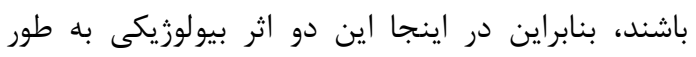

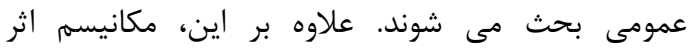

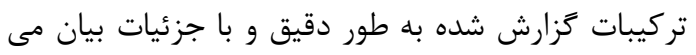
كردد. دو مكانيسم اصلى براى اثرات سيتوتوكسيك و ضدسرطان

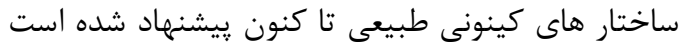

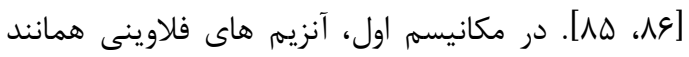

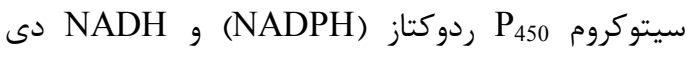

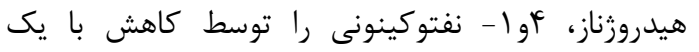

غيرفعال مى باشد [\&/]. ديوسييرن (ع) اولين بار از گياه Diospyros montana Roxb. اين ساختار طى ساليان متمادى همجنين از كونه هاى

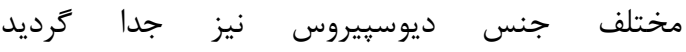

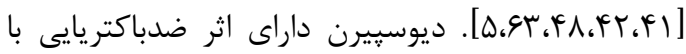

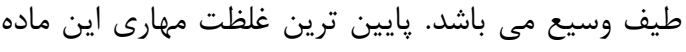

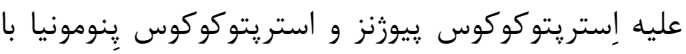

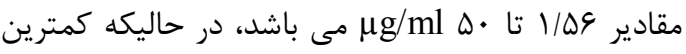

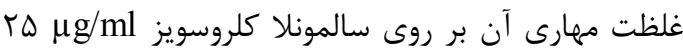

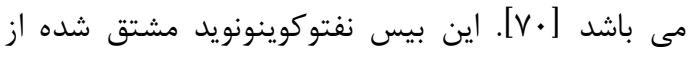
كياه اثر مهارى قابل توجهى نيز بر روى رشد ليشمانيا در



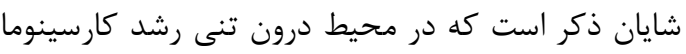

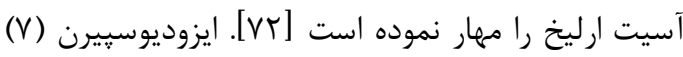

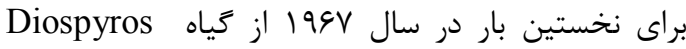
chloroxylon Roxb.

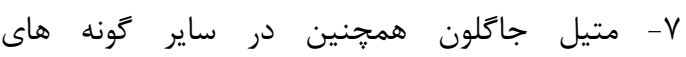

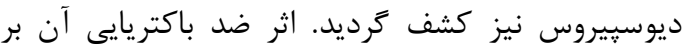
رورى باكترى هاى گرم مثبت بسيار بيشتر از ديوسيرن

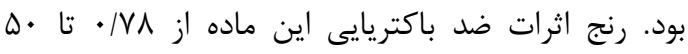

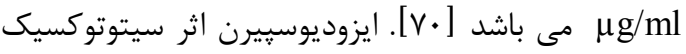
بسيار قوى در محيط برون تنى بر روى رده هاى سلولى بلى Hep-3B

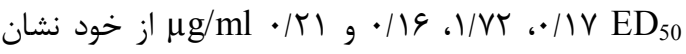
داده است [VD]. بيان اين مطلب قابل ارزش است كه

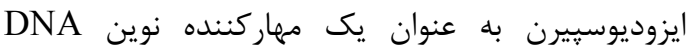

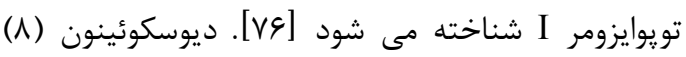
براى اولين بار از D. tricolor جدا كرديد [VV]

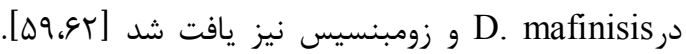

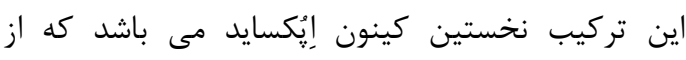

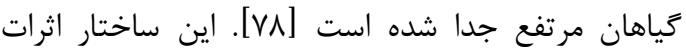
ضدباكتريايى قابل ملاحظه اي بر روى استافيلوكوكوس استي

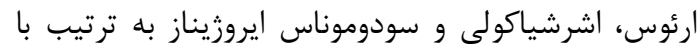

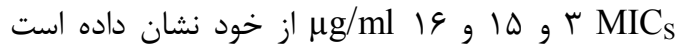

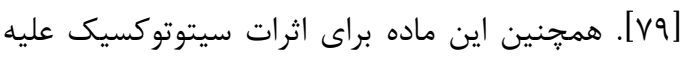

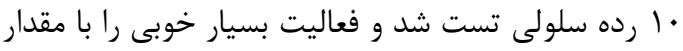

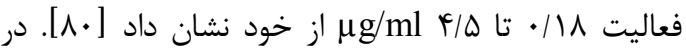


باشد [9V]. ايزوديوسيرن خاصيت سيتوتوكسيك عليه رده هاى سلولى سرطانى از خود نشان داده است. مكانيسم همراه با ايزوديوسيّيرن، مهار مستقيم تويوايزومراز I انسانى آنى



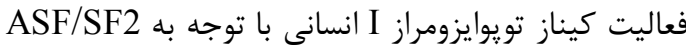

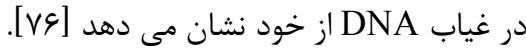
رابطه ساختار ـ اثر تركيبات f و ا ا - نفتوكينونى فارماكوفور أوا- نفتوكينونى به علت دارا بودن اثرات الترات بيولوزيكى مختلفى همجون اثر ضدسرطان، ضدباكترى،

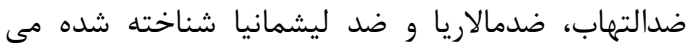

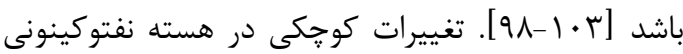
اين ساختار موجب اثراتى بر روى فعاليت هاى بيولوزيك

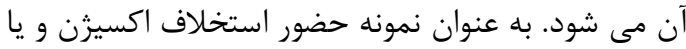

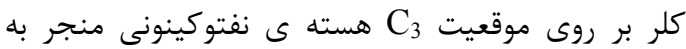

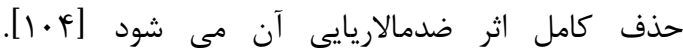
تحقيقات برون تن نشان داده است كه تركيباتى كه در آند

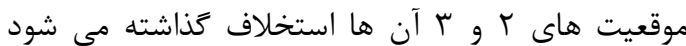

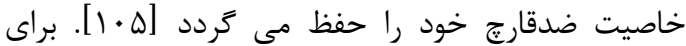

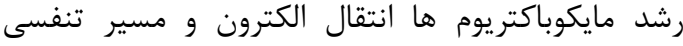

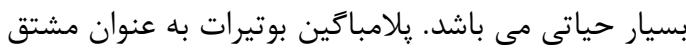


است. اين ماده مصرف اكسيرن را با اثر در انتقال الكترون

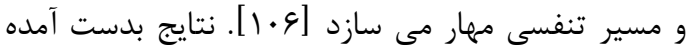
از تست هاى مختلف بيولوزيكى بيان كرده اند كه كتون



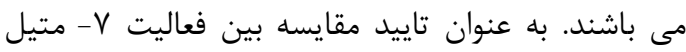

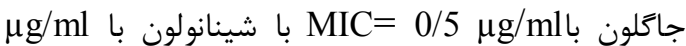

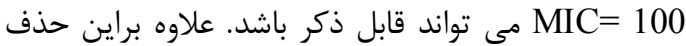

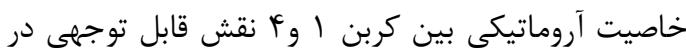

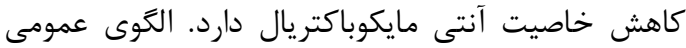
تركيبات بسيار فعال همجون V- متيل جاكلون بسيار شبيه به بخش مسئول انتقال الكترون در مايكوباكتريوم

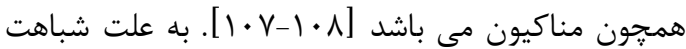
بين ساختار شيميايى V- متيل جاكلون و مناكيون، اين

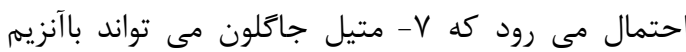
هايى كه در توالى انتقال الكترون مايكوباكتريوم نقش

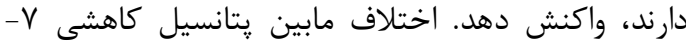

الكترون به سِمى كينون تبديل مى كنند. اين سِمى كينون ها دوباره مى توانند به كينون ها با انتقال الكترون به اكسيرن اكسيد شوند. اين سيكل كاهش و اكسيد به نام سيكل redox شناخته مى شود. دومين مكانيسم وابسته مى باشد به قدرت الكتروفيليسيتى كينون ها كه آنها رانيا قادر مى سازد تا با كروه هاى تيول بروتئين ها و كلوتاتئيون

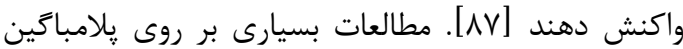
جدا شده از جنس ديوسيّروس انجام كرفته شده است و نتايج نشان مى دهد كه اين ماده مى تواند تركيب راهبر آندان

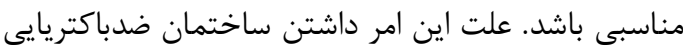

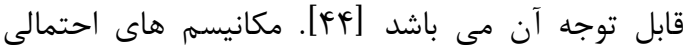

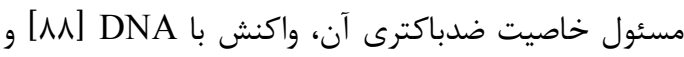
همجنين واكنش هاى اكسيدكنندگى در سلول هایى

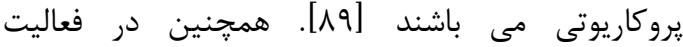
سيتوتوكسيك آن، سيكل redox به عنوان مكانيسم عمل

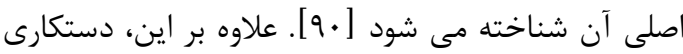

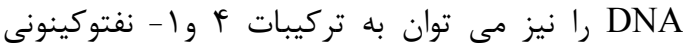

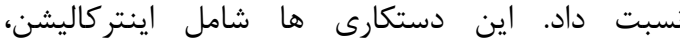
آلكيلاسيون، القاء شكست مارييجى و مهار آنزيم هاى اينتين وابسته به DNA همجيون تويوايزومرزها مى باشئن

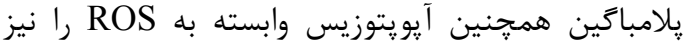
تحريك مى نمايد [91]]. تركيب كارسى فلورون شامل

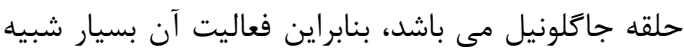

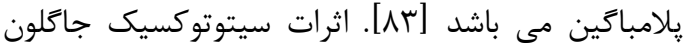
همجنين مى تواند به علت شركت داشتن در كنزو كاسيون

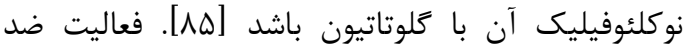
توبر كلوزيس تركيب V- متيل جاتلون احتمال دارد وابسته به مهار مصرف اكسيرن در باكترى مايكوباكتريوم

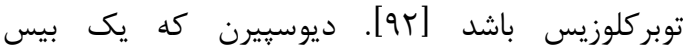
نفتوكوينونويد كياهى مى باشد مى تواند رشد ليشمانيا

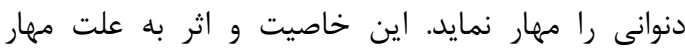
DNA تويرايزومراز تيب I مى باشد [آهو]. مطالعات انجام شده بر روى ديوسييرن نشان داده است كه فعاليت

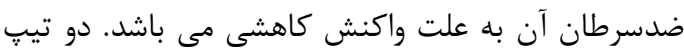

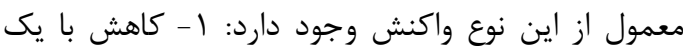

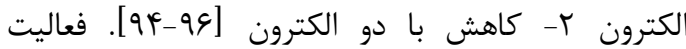
بيولوزيك ديوسييرون به علت كاهش با يك الكترون مى 
[ I l V] ] بنابراين، احتمال دارد جايكزينى مناسب آمينى

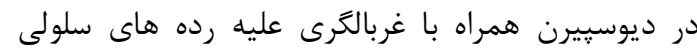

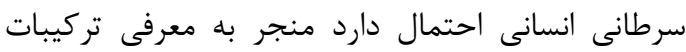
مفيدى در درمان سرطان و تومورهاى بدخيم كردد.

\section{نتيجه كيرى}

اين مقاله مرورى || تركيب f و و ا- نفتوكينونى جدا شده

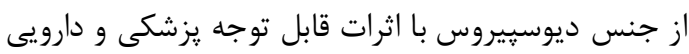

را مورد بحث قرار كرفته است. ما برروى منابع، استفاده

هاى درمانى و اثرات بيولوزيك آن همراه با مكانيسم هاى

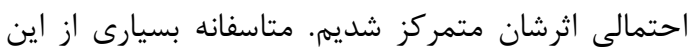

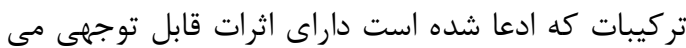

باشند، در محيط درون تنى مورد مطالعه قرار نكرفته اند.

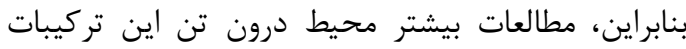

ضرورى مى باشد و تحقيقات سيستماتيك بيشترى بر بر نائر

روى اين دسته از تركيبات غنى مورد نياز مى باشد. لُ و والـ

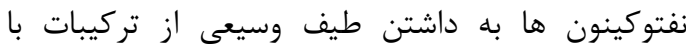
استخلاف هاى مختلف كه بر روى اهداف خاصى در درمان بيمارى هاى مختلف اثر مى كذارند، شناخته شده و معروف مى باشند. به طور خلاصه، اين مقاله مرورى جهت

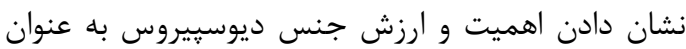
منبع f أ و ا - نفتو كينون ها نعاشته شد.

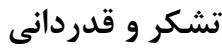

نويسندكان از دانشكاه علوم يزشكى خراسان شمالى و وردان مركز تحقيقات ايمنى فرآورده هاى طبيعى و كياهان

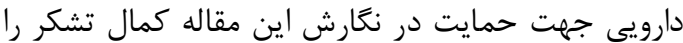

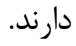

\section{References}

1. Hegnauer R, Chemotaxonomie der Pflanzen, Birkhaiiser, Basel 1996, 18-98.

2. Shastri, BN, Wealth ofIndia-Raw Materials, CSIR Publication, New Delhi 1952, 19-23.

3. Watt JM, Breyer-Brandwijk MBG, Medicinal and Poisonous Plants of Eastern and Southern Africa, Livingston, Edinburgh, 1962.

4. Watt MB, A Dictionary of the Economic Products of India, Cosmo Publication, New Delhi 1956 17-48.

5. Waterman PG, Mbi CN, The sterols and dimeric naphthoquinones of the barks of three West Africa Diospyros species, Planta Medica 1979; 37 (3) 241-246.
متيل جاگلون و مناكيون منجر به توقف جريان الكترون مى گردد. علاوه بر آن، اين ماده مى تواند از جفت شدن شند

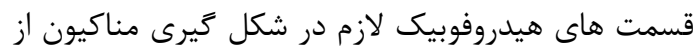
طريق باند شدن به آنزيم مسئول توليد مناكيون، جلوكيرى نمايد [9 • 1]. بنابراين اصلاحاتى در ساختار اين

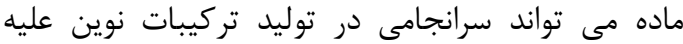
مايكوباكتريوم ها باشد. مطالعات رابطه ساختمان - اثر و واند

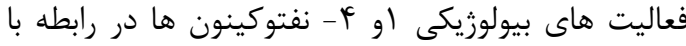

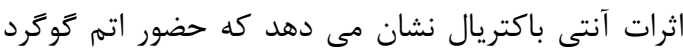

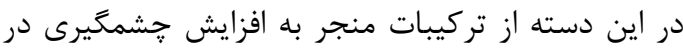

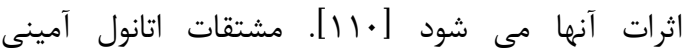
ديوسييرن اثرات بهبودى با ارزشى در فعاليت عليه

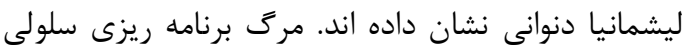
در ليشمانيا بر اثر استرس و استفاده دارويى اتفاق مى افتد

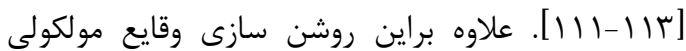

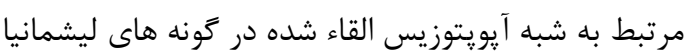
توسط أ و ا - نفتوكينون ها مى تواند به شناخت هدف درمانى جديد در بيمارى ليشمانيا كمك شايانى نمايد.

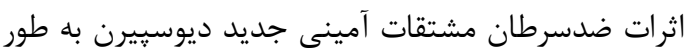
قابل توجهى در محيط درون تن و نيز برون تن پيشرفت

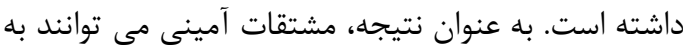

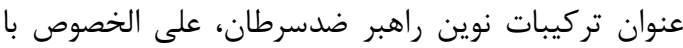


fllf]. قابل ذكر مى باشد كه معرفى استخلاف اتانول

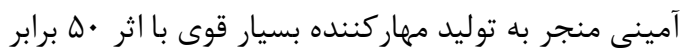

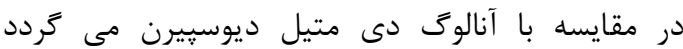

6. Berry PE, Savolainen V, Sytsma KJ, Hall JC, Chase, MW, Lissocarpa is sister to Diospyros (Ebenaceae) 2001; 56(3): 725-729.

7. Duangjai S, Wallnöfer B, Samuel R, Munzinger J, Chase, MW, Generic delimitation and relationships in Ebenaceae sensu lato: Evidence from six plastid DNA regions, American Journal of Botany 2006; 93 (12):1808-1827.

8. Hiern, WP, ISOTYPE. Diospyros subacuta, Trans Camb Phil Soceity 1873; 12, 27-29.

9. Schmidt E, Lötter M, McCleland W, Trees and shrubs of Mpumalanga and Kruger National Park, Jacana, Johannesburg 2002, 140-148.

10. Bailey LH, Manual of Cultivated Plants Most Commonly Grown in the Continental United 
States and Canada, Mcmillan Publishing Company, New York, 1949, 124-154.

11. Chopra RN, Badhwar RL, Ghosh S, Poisonous Plants of India, Government of India Press, Calcutta 1949, 1124-1200.

12. Watt JM, Breyer-Brandwijk MG, The Medicinal and Poisonous Plants of South Africa, Livingstone, Edinburgh 1932, 124-127.

13. Chopra RN, Nayar SL, Chopra IC, Glossary of Indian Medicinal Plants, CSIR, New Delhi $1956,125-128$

14. Wu SC, Yang YH, Hsu KK, Chen FC,Technical Bull Exp Forest, NTU, Taipei 1972, 145-148.

15. Sadun EH, Vajrasthira $\mathrm{S}$, The effect of maklua (Diospyros mollis) in the treatment of human hookworm Parasitology 1954,40:49.

16. Phongboonrod S, Medicinal Plants of Thailand, Kasem Banakit, Bangkok 1979, 12-18.

17. Yan XZ, Kuo YH, Lee TJ, Shih TS, Chen $\mathrm{CH}$, McPhail DR, McPhail AT, Lee KH, Phytochemistry 1989; 28 (1):1541-1548

18. Jains SK, Dictionary of Indian Folk Medicine and Ethanobotany, Deep Publication, New Delhi 1991, 1742-1765.

19. Chatterjee A, Pakrashi SC, The Treatise on Indian Medicinal Plants, Publication and Information Directorate, New Delhi 1995, 125 129.

20. Mallavadhani UV, Panda AK, Rao YR, Pharmacology and chemotaxonomy of Diospyros Phytochemistry 1998; 49 (4): 901-951

21. Kirtikar KR, Basu BD, Indian Medicinal Plants, Allahabad, India 1933, 121-124.

22. Khan MR, Nkunya, MHH, Wevers H, Triterpenoids from leaves of diospyros species, Planta Medica 1980; 38 (4): 380-381.

23. Gafner F, Chapuis JC, Msonthi D, Hostettmann J, Cytotoxic naphthoquinones, molluscicidal saponins and flavonols from Diospyros zombensis, Phytochemistry 1987; 26 (9):2501-2503.

24. Burkhill IH, A Dictionary of the Economic Products of the Malay Peninsula, Ministry of Agriculture and Co-op, Kuala Lumpur, 1966, 110.

25. Z. Sasakawa, J Jpn Soc Intern Med. 43,858 (1955).

26. Uhe G, Medicinal plants of samoa - A preliminary survey of the use of plants for medicinal purposes in the Samoan Islands, Economic Botany 1974; 28 (1):1-30.

27. Buckpitt A, Boland B, Isbell M, Morin D, Shultz M, Baldwin R, Plopper C, Naphthalene-induced respiratory tract toxicity:
Metabolic mechanisms of toxicity , Drug Metabolism Reviews 2002; 34 (4):791-820.

28. Benz CC, Atsriku C, Yau C, Britton D, Schilling B, Gibson BW, Baldwin MA, Scott GK, Novel pathways associated with quinoneinduced stress in breast cancer cells Drug Metabolism Reviews 2006; 38 (4): 601-613.

29. Lagorio SH, Bianchi DA, Sutich EG, Kaufman TS, Synthesis and antimicrobial activity of pyranobenzoquinones related to the pyranonaphthoquinone antibiotics, European Journal of Medicinal Chemistry 2006; 41 (11):1333-1338.

30. Norinder U, In silico modelling of admet - A minireview of work from 2000 to 2004, SAR and QSAR in Environmental Research 2005; 16 (12):1-11.

31. Alireza Nematollahi, Noushin Aminimoghadamfarouj, Christophe Wiart,Design and Modeling Studies on Liriodenine derivatives as novel topoisomerase II inhibitors, International Journal of ChemTech Research 2011, Vol.3, No.3,1622-1627[Persian]

32. Alireza Nematollahi, Noushin Aminimoghadamfarouj, Mohammad Reza Jalilvand, Seyed Ali Vakili, Design and Molecular Docking Studies of luteolin derivatives, from Biebersteinia multifida DC., as novel HMG-CoA reductase inhibitors, International Journal of ChemTech Research 2012; Vol.4, No.2, pp 733-738[Persian]

33. Alireza Nematollahi, Noushin Aminimoghadamfarouj, Mohammad Reza Jalilvand, Bahram Bibak, Seyed Ali Vakili In Silico Molecular Modeling and Docking Studies on Luteolin Derivatives as Novel Helicobacter Pylori $\beta$-hydroxyacyl-acyl carrier protein dehydratase Inhibitor from Biebersteinia multifida DC., International Journal of ChemTech Research, ,2012, Vol.4, No.2, pp $767-$ 774[Persian]

34. Akak CM, Djama CM, Nkengfack AE, Tu PF, Lei LD, New coumarin glycosides from the leaves of Diospyros crassiflora (Hiern) Fitoterapia 2010; 81 (7): 873-877.

35. Schoonhoven LM, Van Loon JJA, Dicke M, Insect-plant biology, Routledge, New York 1997, 32-39.

36. Ohigashi H, Takamura H, Koshimizu K, Search for possible antitumor promoters by inhibition of 12-O-tetradecanoylphorbol-13acetate-induced Epstein-Barr virus activation; ursolic acid and oleanolic acid from an antiinflammatory Chinese medicinal plant, 
Glechoma hederaceae L. Cancer Letters1986; 30 (2):143-151.

37. Vijver VD, Distribution of plumbagin in the mplumbaginaceae, Phytochemistry 1972; 11 (11): 3247-3248.

38. Herath WHMW, Rajasekera NDS, Sultanbawa S, Wannigama MU, Balasubramaniam S, Triterpenoid, coumarin and quinone constituents of eleven Diospyros species (Ebenaceae) Phytochemistry 1978; 17 (5):10071009.

39. Hirota M, Mori $T$, Yoshida M , Iriye R, Suppression of tumor promoter-induced inflammation of mouse ear by ursolic acid and 4,4-dimethylcholestane derivatives. Agric. Biol. Chem. 1990, 54(4), 1073-1075.

40. Kaul R, Ray A.C. \& Dutt S, Constitution of the active principle of Embelia ribes

Pt.1, J . Indian Chem. Soc. 1929, 6, 577-586.

41. Schön K. Studies on carotenoids: The carotenoids of Diospyros fruits. II. The carotenoids of arbutus fruits (Arbutus unedo), Biochem J 1935, 29:1779.

42. RG Cooke and H Dowd, Colouring Matters of Australian Plants. II. Naphthoquinones from Diospyros hebecarpa A. Cunn, Australian Journal of Scientific Research 1952; 5(4) 760 767

43. Khan MR, Rwekika E, Triterpenoids from the leaves of four species of family ebenaceae, Fitoterapia 1992; 63 (4): 375-376.

44. Evans PH, Bowers WS, Litaudon M, Sevenet T, Plumbagin from Diospyros olen, Molecules 1999; 4 (4):93-100.

45. Hazra B, Sarkar R, Bhattacharyya S, Ghosh PK, Chel G, Dinda B, Synthesis of plumbagin derivatives and their inhibitory activities against Ehrlich ascites carcinoma in vivo and Leishmania donovani promastigotes in vitro, Phytotherapy Research 2002;16 (2):133-137.

46. Chandra S, Sastry MS, Chemical examination of Diospyros cordifolia Roxb, Indian Journal of Pharmaceutical Sciences 1989; 51 (6):258-259.

47. Jain N, Yadava R, Peregrinol A, Lupane type triterpene from the fruits of Diospyros peregrina, Phytochemistry 1994: 35 (4):10701072 .

48. T.J. Lillie, O.C. Musgrave, and D. Skoyles, J Chem Soc. 20, 2155 (1976).

49. Sundararamaiah T, Ramraj SK, Lakshman Rao K, Vimalabai V, Isolation of lupeol group of triterpenes from Dillenia indica Linn. and Diospyros perigrina, Journal of the Indian Chemical Society 1976; 53 (6): 638-645.
50. Yoshihira K, Tezuka M, Natori S, Naphthoquinone derivatives from diospyros SPP: bisisodiospyrin, a tetrameric naphthoquinone, Tetrahedron Letters 1970; 11(1):7-10.

51. Davy G.S., Halsall T.G., Jones E.R.H., Meakins, G.D. The chemistry of the triterpenes, Part X,

The structures of some isomerisation products from betulin and betulinic acid. J. Chem. Soc.

$1951 ; 2702-2705$.

52. Misra G, Nigam SK, Mitra CR, Steroids and triterpenoids of Diospyros montana, Phytochemistry 1972; 11 (4):1508-1509.

53. Dutta PK, Dutta NL, Chakravarti RN, Sterols and triterpenes of Diospyros montana, Phytochemistry 1972; 11 (3):1180-1181.

54. Tiwari KP, Masood M, Minocha PK, Chemical constituents ofGemlina phillipinensis, Adenocalymna nitida, Allamanda cathartica,Averrhoa carambola and Maba buxifolia, J Indian Chem Soc1979;56:944.

55. Hasbun C, Carballo J, Castro O, Poveda L (1988) Major Constituents of the Wood of Diospyros-Nicaraguensis, Fitoterapia 1988; 59, 157.

56. R.H. Thomson, Naturally Occurring Quinones, 2nd edn. (Academic Press, London, 1971).

57. Salae AW, Karalai C, Ponglimanont C, Kanjana-Opas A, Yuenyongsawad S, Naphthalene derivatives from Diospyros wallichii, Canadian Journal of Chemistry 2010; 88 (9): 922-927.

58. L.R. Rao, C.S. Rao, and T. SundarRamaiah, The Chemical Examination of Diospyros Species, Curr Sci. 1966 35, 457-58.

59. A.G. Brown and R.H. Thomson, Ebenaceae extractives. Part II. Naphthaldehydes from Diospyros ebenum koen, J Chem Soc. 1965,4292 .

60. S. Matsuura, K. Asano, K. Oba, and M. Mizuno, J Pharm Soc Jap. 91, 905 (1971).

61. Jeffreys JAD, Bin Zakaria M, Waterman PG, 3'-methoxydiospyrin, a 7-methyljuglone dimer from diospyros mannii Phytochemistry 1983; $22(8): 1832-1833$.

62. Gupta RK, Chandra S, Mahandevan V, Chemical composition of Sphaeranthus indicus Linn, Indian J Pharm 1967;29:47-8.

63. A.V.B Sankaram and G.S. Sidhu, Indian J Chem. 2,467 (1964).

64. Mahapatra A, Mativandlela SPN, Binneman B, Fourie PB, Hamilton CJ, Meyer JJM, Van der Kooy F, Lall N, Activity of 7-methyljuglone derivatives against Mycobacterium tuberculosis 
and as subversive substrates for mycothiol disulfide reductase , Bioorganic and Medicinal Chemistry 2007 ; 15 (24):7638-7646.

65. Carter FL, Garlo AM, Stanley JB, Termiticidal components of wood extracts: 7Methyljuglone from Diospyros virginiana, Journal of Agricultural and Food Chemistry 1978; 26 (4): 869-873.

66. Weigenand O, Hussein AA, Lall N, Meyer JJM, Antibacterial activity of naphthoquinones and triterpenoids from Euclea natalensis root bark, Journal of Natural Products 2004; 67 (11):1936-1938.

67. R.K. Gupta and R.D. Tiwari, Proc Nat Acad Sci India. 34, 180 (1964).

68. Gu JQ, Graf TN, Lee D, Chai, HB, Mi Q, Kardono, LBS, Setyowati FM, Oberlies NH, Cytotoxic and antimicrobial constituents of the bark of Diospyros maritima collected in two geographical locations in Indonesia, Journal of Natural Products 2004; 67 (7):1156-1161.

69. Didier A, Modern Arene Chemistry, WileyVCH Verlag GmbH, Weinheim 2002, 120201208.

70. Adeniyi BA, Fong HHS, Pezzuto JM, Luyengi L, Odelola HA, Antibacterial activity of diospyrin, isodiospyrin and bisisodiospyrin from the root of Diospyros piscatoria (Gurke) (Ebenaceae), Phytotherapy Research 2000; 14 (2):112-117.

71. Cordell GA, Review article number 109: Changing strategies in natural products chemistry, Phytochemistry 1995; 40 (6):15851612.

72. Pisha E, Chai H, Lee IS, Chagwedera TE, Farnsworth NR, Cordell GA, Beecher CWW, Pezzuto JM, Discovery of betulinic acid as a selective inhibitor of human melanoma that functions by induction of apoptosis, Nature Medicine 1995; 1 (10):1046-1051.

73. Sidhu GS, Prasad KK, isodiospyrin - a novel binaphthaquinone showing atropisomerism and Other extractives from diospyros chloroxylon, Tetrahedron

Letters 1967; 8 (30):2905-2910.

74. A.L. Fallas, R.H. Thomson, Binaphthaquinones from Diospyros species., J Chem Soc., 1968, 2279-82.

75. Kuo YH, Chang CI, Li SY, Chou CJ, Chen $\mathrm{CF}$, Kuo YH, Lee KH, Cytotoxic constituents from the stems of Diospyros maritima, Planta Medica 1997; 63 (4):363-365.

76. Ting CY, Hsu CT, Hsu HT, Su JS, Chen, TY, Tarn WY, Kuo YH, Hwang J, Isodiospyrin as a novel human DNA topoisomerase I inhibitor, Biochemical Pharmacology2003; 66 (10):1981-1991.

77. G.S. Sidhu, A.V.B. Sankaram and S. Mahmood, Extractives from Diospyros species: Part III -

New naphthoquinones and naphthols from the heartwood of Diospyros melanoxylon Roxb., Indian J Chem. 1968, 6,681-91.

78. T.J. Lillie and O.C. Musgrave, Ebenaceae extractives. Part 8 . The structure of diosquinone and reactions of related quinone epoxides, J. Chem. Soc., Perkin Trans. 1, 1980, 1161-1164.

79. Lajubutu BA, Pinney RJ, Roberts MF, Odelola HA, Oso BA, Antibacterial activity of diosquinone and plumbagin from the root of Diospyros mespiliformis (Hostch) (Ebenaceae), Phytotherapy Research 1995; 9 (5):346-350.

80. Adeniyi BA, Robert MF, Chai H, Fong HHS, In vitro cytotoxicity activity of diosquinone, a naphthoquinone epoxide Phytotherapy Research 2003; 17 (3):282-284.

81. Tangmouo JG, Meli AL, Komguem J, Kuete V, Ngounou FN, Lontsi D, Beng VP, Sondengam BL, Crassiflorone, a new naphthoquinone from Diospyros crassiflora (Hien) Tetrahedron Letters 2006; 47 (18):3067-3070.

82. Uete V, Tangmouo JG, Marion Meyer JJ, Lall N, Diospyrone, crassiflorone and plumbagin: three antimycobacterial and antigonorrhoeal naphthoquinones from two Diospyros spp, International Journal of Antimicrobial Agents 2009; 34 (4):322-325.

83. Ganapaty S, Steve Thomas P, Karagianis G, Waterman PG, Brun R, Antiprotozoal and cytotoxic naphthalene derivatives from Diospyros assimilis, Phytochemistry 2006; 67 (17):1950-1956.

84. prajoubklang A, Sirithunyalug B, Charoenchai P, Suvannakad R, Sriubolmas N, Piyamongkol S, Kongsaeree P, Kittakoop P, Bioactive deoxypreussomerins and dimeric naphthoquinones from Diospyros ehretioides fruits: Deoxypreussomerins may not be plant metabolites but may be from fungal epiphytes or endophytes, Chemistry and Biodiversity 2005; 2 (10):1358-1367.

85. Inbaraj JJ, Chignell CF, Cytotoxic Action of Juglone and Plumbagin: A Mechanistic Study Using HaCaT Keratinocytes, Chemical Research in Toxicology 2004; 17 (1):55-62.

86. Castro FAV, Mariani D, Panek AD, Eleutherio E.C.A., Pereira, M.D. Cytotoxicity mechanism of two naphthoquinones 
(menadione and plumbagin) in Saccharomyces cerevisiae 2008; 3 (12), art. no. e3999.

87. Eilenberg H, Pnini-Cohen S, Rahamim Y, Sionov E, Segal E, Carmeli S, Zilberstein A, Induced production of antifungal naphthoquinones in the pitchers of the carnivorous plant Nepenthes khasiana, Journal of Experimental Botany 2010; 61 (3):911-922.

88. Demma J, Hallberg K, Hellman B, Genotoxicity of plumbagin and its effects on catechol and NQNO-induced DNA damage in mouse lymphoma cells, Toxicology in Vitro 2009; 23 (2):266-271.

89. Archibald FS, Fridovich I, Manganese, superoxide dismutase, and oxygen tolerance in some lactic acid bacteria, Journal of Bacteriology 1981; 146 (3): 928-936.

90. Sandur SK, Ichikawa H, Sethi G, Kwang SA, Aggarwal BB, Plumbagin (5-hydroxy-2methyl-1,4-naphthoquinone) suppresses NF- $\mathrm{B}$ activation and $\mathrm{NF}-\kappa \mathrm{B}-$ regulated gene products through modulation of $\mathrm{p} 65$ and $\mathrm{I} \kappa \mathrm{B} \alpha$ kinase activation, leading to potentiation of apoptosis induced by cytokine and chemotherapeutic agents, Journal of Biological Chemistry 2006; 281 (25):17023-17033.

91. Miguel del Corral JM, Castro MA, Oliveira AB, Gualberto SA, Cuevas C, Feliciano AS, New cytotoxic furoquinones obtained from terpenyl-1,4-naphthoquinones and 1,4anthracenediones, Bioorganic and Medicinal Chemistry2006; 14 (21):7231-7240.

92. Anabwani G, Canfield CJ, Hutchinson DBA, Combination atovaquone and proguanil hydrochloride vs. halofantrine for treatment of acute Plasmodium falciparum malaria in children, Pediatric Infectious Disease Journal1999; 18 (5):456-461.

93. RayS, Hazra B, Mittra B, Das A, Majumder HK, Diospyrin, a bisnaphthoquinone: A novel inhibitor of type I DNA topoisomerase of Leishmania donovani, Molecular Pharmacology 1998; 54 (6):994-999.

94. De Abreu FC, De Ferraz PAL, Goulart MOF, Some applications of electrochemistry in biomedical chemistry. Emphasis on the correlation of electrochemical and bioactive properties, Journal of the Brazilian Chemical Society 2002; 13 (1):19-35.

95. Hillard EA, De Abreu FC, Ferreira DCM, Jaouen G, Goulart MOF, Amatore C, Electrochemical parameters and techniques in drug development, with an emphasis on quinones and related compounds, Chemical Communications 2008; (23):2612-2628.
96. Pinto AV, De Castro SL, The trypanocidal activity of naphthoquinones: A review, Molecules 2009; 14 (11):4570-4590.

97. Norhanom AW, Hazra B, Inhibition of tumour promoter-induced epstein-barr virus activation by diospyrin, a plant-derived antitumour compound, and its synthetic derivatives, Phytotherapy

Research 1997;11 (8):588-590.

98. Lee EJ, Lee HJ, Park HJ, Min HY, Suh ME, Chung HJ, Lee SK, Induction of G 2/M cell cycle arrest and apoptosis by a benz f.indole-4,9dione analog in cultured human lung (A549) cancer cells, Bioorganic and Medicinal Chemistry Letters 2004;14 (20):5175-5178.

99. Song GY, Kim Y, Zheng XG, You YJ, Cho H, Chung JH, Sok DE, Ahn BZ, Naphthazarin derivatives (IV): Synthesis, inhibition of DNA topoisomerase I and cytotoxicity of 2- or 6-acyl5,8-dimethoxy-1,4- naphthoquinones, European Journal of Medicinal Chemistry 2000;35 (3):291298.

100. Tran T, Saheba E, Arcerio AV, Chavez V, Li QY, Martinez LE, Primm TP, Quinones as antimycobacterial agents Bioorganic and Medicinal Chemistry 2004; 12 (18):4809-4813.

101. Lien JC, Huang LJ, Wang JP, Teng CM, Lee KH., Kou SC, Synthesis and antiplatelet, antiinflammatory, and antiallergic activities of 2-substituted 3-chloro-1,4naphthoquinone derivatives, Bioorganic and Medicinal Chemistry 1997; 5(12):2111-2120.

102. Dos Santos EVM, Carneiro JWDM, Ferreira VF, Quantitative structureactivity relationship in aziridinyl-1,4naphthoquinone antimalarials: Study of theoretical correlations by the PM3 method, Bioorganic and Medicinal Chemistry 2004;12 (1):87-93.

103. Valderrama JA, Benites,J, Cortés M, Pessoa-Mahana H, Prina E, Fournet A, Studies on quinones. Part 38: Synthesis and leishmanicidal activity of sesquiterpene 1,4quinones, Bioorganic and Medicinal Chemistry 2003;11 (22):4713-4718.

104. Likhitwitayawuid K, Kaewamatawong R, Ruangrungsi N, Krungkrai J, Antimalarial naphthoquinones from Nepenthes thorelii, Planta Medica 1998; 64 (3):237-241.

105. Tandon VK, Maurya HK, Tripathi A, ShivaKeshava GB, Shukla PK, Srivastava P, Panda D, 2,3-Disubstituted-1,4naphthoquinones, 12H-benzo b.phenothiazine6,11-diones and related compounds: Synthesis and Biological evaluation as potential 
antiproliferative and antifungal agents, European Journal of Medicinal Chemistry 2009; 44 (3):1086-1092.

106. Mathew R, Kruthiventi AK, Prasad JV, Kumar SP, Srinu G, Chatterji D, Inhibition of mycobacterial growth by plumbagin derivatives, Chemical Biology and Drug Design 2010; 76 (1):34-42.

107. Kooy VD, Meyer JJM, Lall N, Antimycobacterial activity and possible mode of action of newly isolated neodiospyrin and other naphthoquinones from Euclea natalensis South African Journal of Botany 2006; 72 (3):349-352.

108. Garbe TR, Co-induction of methyltransferase Rv0560c by naphthoquinones and fibric acids suggests attenuation of isoprenoid quinone action in Mycobacterium tuberculosis, Canadian Journal of Microbiology 2004; 50 (10)771-778.

109. Truglio JJ, Theis K, Feng Y, Gajda R, Machutta C, Tonge PJ, Kisker C, Crystal Structure of Mycobacterium tuberculosis MenB, a Key Enzyme in Vitamin $\mathrm{K}_{2}$ Biosynthesis, Journal of Biological Chemistry 2003; 278 (43):42352-42360.

110. Tandon VK, Yadav DB, Singh RV, Vaish M, Chaturvedi AK, Shukla PK, Synthesis and biological evaluation of novel 1,4naphthoquinone derivatives as antibacterial and antiviral agents, Bioorganic and Medicinal Chemistry Letters 2005;15 (14):3463-3466.

111. Debrabant A, Lee N, Bertholet S, Duncan R, Nakhasi HL, Programmed cell death in trypanosomatids and other unicellular organisms, International Journal for Parasitology2003; 33 (3):257-267.
112. Sen N, Das BB, Ganguly A, Mukherjee T, Tripathi G, Bandyopadhyay S, Rakshit S, Majumder HK, Camptothecin induced mitochondrial dysfunction leading to programmed cell death in unicellular hemoflagellate Leishmania donovani, Cell Death and Differentiation 2004;11 (8): 924-936.

113. Paris C, Loiseau PM, Bories C, Bréard J, Miltefosine Induces Apoptosis-Like Death in Leishmania donovani Promastigotes, Antimicrobial Agents and Chemotherapy 2004; 48 (3): 852-859.

114. Chakrabarty S, Roy M, Hazra $\mathrm{B}$, Bhattacharya RK, Induction of apoptosis in human cancer cell lines by diospyrin, a plantderived bisnaphthoquinonoid, and its synthetic derivatives, Cancer Letters 2002;188 (1-2): 8593.

115. Hazra B, Das Sarma M, Kumar B, Basu S, Das K, Pandey BN, Mishra KP, Cytotoxicity of diospyrin and its derivatives in relation to the generation of reactive oxygen species in tumour cells in vitroandin vivo, Chemotherapy 2007; 53 (3):173-176.

116. Kumar B, Joshi J, Kumar A, Pandey BN, Hazra B, Mishra KP, Radiosensitization by diospyrin diethylether in MCF-7 breast carcinoma cell line, Molecular and Cellular Biochemistry 2007; 304 (1-2): 287-296.

117. Das Sarma M, Ghosh R, Patra A, Hazra B, Synthesis and antiproliferative activity of some novel derivatives of diospyrin, a plant-derived naphthoquinonoid, Bioorganic and Medicinal Chemistry 2007; 15 (11): 3672-3677. 
Review Article

\title{
Reviews On 1,4-naphthoquinones from Diospyros L
}

\author{
Bibak $B^{1}$, Kamali $H^{2}$, Nematollahi $A^{3 *}$ \\ ${ }^{1}$ Assistant Professor of Physiology, School of Medicine, North Khorasan University of Medical Sciences, \\ Bojnurd, Iran \\ ${ }^{2}$ M.Sc of Chemical Engineering, Research Center of Natural Products Safety and Medicinal Plants, North \\ Khorasan University of Medical Sciences, Bojnurd, Iran \\ ${ }^{3}$ Pharm.D, Research Center of Natural Products Safety and Medicinal Plants, North Khorasan University of \\ Medical Sciences, Bojnurd, Iran
}

*Correspondence Author:

Natural Products Safety and

Medicinal Plants Research

Center, North Khorasan

University of Medical Sciences

Email:

alireza_nemat@yahoo.com

\begin{abstract}
Background \& Objectives:The term natural products refers to secondary metabolites, small molecules (mol wt $<2000$ amu) produced by an organism such as medicinal plants. These compounds are known as a powerful source to design novel medicines. In this review article one group of the most famous and applicable compounds in the genus Diospyros named 1, 4naphthoquinones is discussed. The aim of this review is to disclose advances in biological, pharmacological researches and phytochemistry of 1, 4- naphthoquinones isolated from this genus, as well as the suggested mechanisms regarding their activities.

Material and Methods: This research was done based on the searching the keywords including Diospyros; 1, 4naphthoquinone; antibacterial; anticancer; Ebnaceae, mechanism through the well-known databases from beginning to 2011.

Results:During the past 60 years many compounds have been isolated from Diospyros $L$. Twelve considerable bioactive structures are reported in this review. plumbagin and diospyrin are two main basic 1, 4-naphthoquinone skeletons, which have been studied extensively in clinical and pharmacological researches. At present the potential for development of leads from 1, 4-naphthoquinones obtained from Diospyros $L$. is growing dramatically, mainly in the area of anticancer and antibacterial investigations. The data prepared and described here is intended to be served as a reference tool to the natural products and chemistry specialists in order to expand the rational drug design
\end{abstract}

Keywords: Diospyros; 1, 4-naphthoquinone; antibacterial; anticancer; Ebnaceae, mechanism

Submitted:24 Oct 2012

Revised:6 Nov 2012

Accepted:27 Nov 2012 